\title{
Application of Genetic Engineering Techniques in the Development of Complex Electromechanical Structures for Marine Robotics
}

\author{
N. M. Shaitor, B. A. Yakimovich, Yu. I. Ryaskov, A. V. Gorpinchenko
}

The current trends in the creation of underwater robotic devices for the study of the world ocean are considered. It is noted that complex types of motion of various effectors cannot be implemented by means of simple electromechanical transducers. Therefore, the use of genetic engineering techniques to create a variety of electromechanical structures that can provide effective functionality of marine robotics objects solves a scientific problem that is of much current interest.

The aim of this work is to analyze and study the possibility of applying the principles of genetic synthesis for the development of new types of energy-efficient transducers built on the basis of complex electromechanical structures and demanded by a wide range of the developing marine robotics.

It is shown how complex electromechanical structures that satisfy a given target function are obtained using genetic operators, together with operators of geometric transformations. The use of genetic engineering techniques for the development of electromechanical transducers expands the possibilities of creating and studying the structural diversity of mechanisms with complex movement of effectors. The configuration of a wave fin propulsion device based on the inductor electromechanical transducer of axial-radial configuration is considered.

A mathematical model, methods and a program for calculating an electromechanical transducer with complex structural axial-radial configuration is developed. The problem of determining the optimal copper/steel ratio is solved, the leading dimensions are obtained and the rational relations of the parameters for calculation of the axial-radial configuration transducer with a minimum mass are established.

Received October 02, 2019

Accepted January 11, 2020

\author{
Nikolay M. Shaitor \\ shaytor1950@mail.ru \\ Boris A. Yakimovich \\ yakimovich52@gmail.com \\ Yury I. Ryaskov \\ rjaskoff@mail.ru \\ Alexander V. Gorpinchenko \\ algornew@mail.ru \\ Sevastopol State University \\ ul. Universitetskaya 33, Sevastopol, 299053 Russia
}


Calculations show that, in comparison with the traditional inductor machines with drumtype rotors, the mass of the active electromagnetic core of axial-radial type transducers can be reduced by a factor of 2.7 to 3.4 with a double reduction in volume. Axial-radial configurations of electromechanical structures designed for electric frequencies of 50 to $400 \mathrm{~Hz}$ are suitable for use in low-speed drives of wind and hydroelectric power plants and propulsion systems of underwater vehicles, and those designed for 1000 to $10000 \mathrm{~Hz}$ can be used in high-speed drives of autonomous power plants of vehicles, aircraft, gyromotors, gas and steam turbines.

Keywords: underwater vehicles, electromechanical structure, genetic procedure, electromagnetic chromosome, axial-radial configuration, wave propulsion device, electromagnetic core, calculation method

\section{Introduction}

At present, technologically advanced countries give considerable attention to developing underwater vehicles and robotic devices. Developers show particular interest in the propulsion system of hydrocoles - marine animals and fish. Their study contributes to the creation of new types of power generating systems intended for development of the world ocean and possessing high propulsive qualities, noiselessness and maneuverability. Most hydrocoles have developed the ability to swim in a wave-like manner. This motion mode is used in some technical models, in particular, highly maneuverable amphibian robots, which are capable of implementing several motion modes used by animals, and include swimming, crawling and sliding [1-4].

In most cases the power technology of autonomous biotechnical models is based on the transformation of electric energy into mechanical energy of effectors [5-7].

However, complex types of motion of the effectors cannot be implemented by means of simple electromechanical transducers. Therefore, the application of genetic engineering techniques for obtaining, analyzing and choosing electromechanical structures capable of ensuring an efficient function of marine robotics objects solves the problem of interest.

The purpose of this paper is to analyze and explore the possibilities of applying the principles of genetic synthesis for the development of new types of energetically efficient electromechanical transducers constructed on the basis of complex electromechanical structures and demanded by a wide range of the developing marine robotics.

In the opinion of the authors of the genetic conception of construction and development of electromechanical structures, the fundamental notion of the primary source of an electromagnetic field (a parental electromagnetic chromosome) and a genetic code is the basis of genetic engineering techniques. Electromechanical structures of the highest level are obtained from parental chromosomes by replicating them. The presence of correspondence between the groups of genetic and geometric operators allows one to formalize the genetic procedures of formation of complex electromechanical structures (Fig. 1). This is achieved by spatial superposition, parallel shift, rotations, translations, combinatory rearrangements, spatial deformations and other algorithmic transformations [8-11].

\section{Application of methods of genetic engineering for development of complex electromechanical structures}

Figure 1 shows possible ways of generating electromechanical structures with symbols in genetic codes. The first two letters of the genetic code encode the geometric shape, and the other 


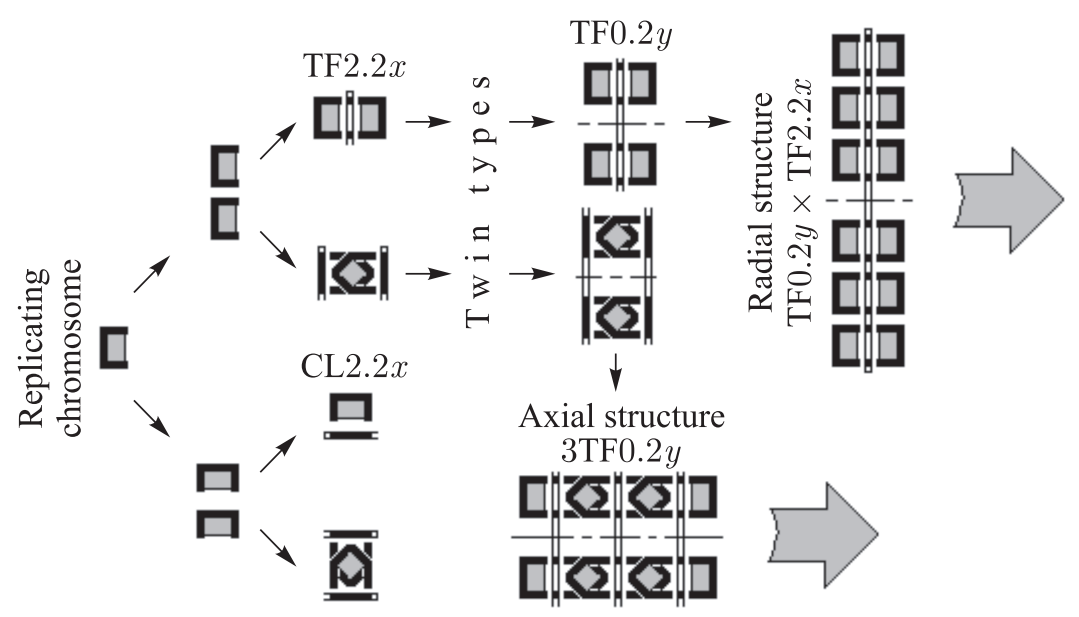

Fig. 1. Generation of complex electromechanical structures based on generating chromosomes.

letters encode the corresponding form of orientation of the primary source of the magnetic field generated by a structure that is a real equivalent of an electromagnetic chromosome. Symbol $X$ means that the direction of field propagation coincides with the main element of the spatial symmetry of the source, whereas symbol $Y$ indicates the orientation of field propagation in the orthogonal direction. The numerical part of the code indicates the number of ruptures (end effects) of the wave of the field in the direction of its propagation (the first digit of the code) and in the orthogonal direction (the second digit). The replications and combinations of the spatial arrangement of the electromagnetic chromosomes allow one to obtain both toroidal flat TF2.2x and cylindrical CL2.2x field sources - generating chromosomes (Fig. 1). The rotation of the graphic primitive element relative to the axis gives chromosomes TF0.2y; the subsequent axial or coaxial arrangement of these chromosomes forms the level of axial 3TF0.2y and radial $($ TF $0.2 y) \times($ TF $2.2 x)$ chromosome complements. From the resulting set one chooses chromosome complements satisfying the prescribed target function.

Further techniques of genetic synthesis of electromechanical structures with radial and axial structures are generated by means of genetic operators of crossing (hybridization), superposition and electromagnetic mutation based on the principles of inversion $[12,13]$.

The axial-radial configuration (ARC) of electromechanical structures can be constructed on the basis of a first ionization potential with toroidal spatial surfaces $($ TF0.0Y $) \times($ TF2.0X), which have been obtained by crossing and possess an extended performance tolerance (Fig. 2).

The construction of the final structural composition $3(\mathrm{TF} 0.0 Y) \times(\mathrm{TF} 2.0 \mathrm{X})$ is achieved by superposition of radial hybride structures 1, 2 and 3 placed on the common axis of rotation. Such a transducer was called the electromechanical transducer of axial-radial configuration [14-17].

When a wave fin propulsion device is mounted inside the cylindrical shell, such a transducer is capable of generating undulatory motion of elastic fin elements (Fig. 3).

A traveling wave is achieved by reciprocating rotation of the disk rotors 1,2 and 3 mounted on the principal axis of the shell in the case of their axial arrangement. The force from the disk rotors is transmitted to the auxiliary axes rigidly attached on the surfaces of the rotors, and from the auxiliary axes to the elastic fin elements mounted on the above-mentioned axes with the possibility of rotation. The phase displacement of the crest of the wave traveling along the principal axis is achieved by the relative displacement of the disk rotors, which undergo 


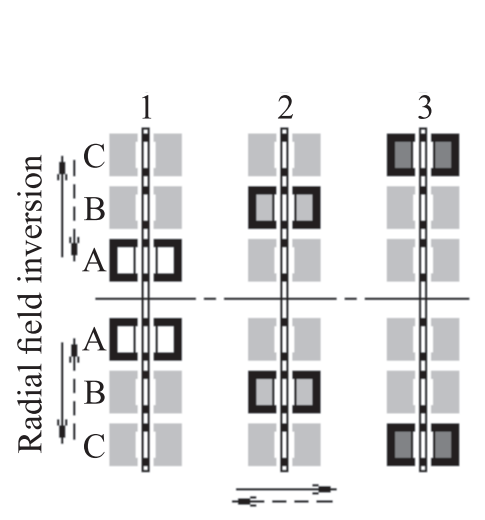

Axial field inversion

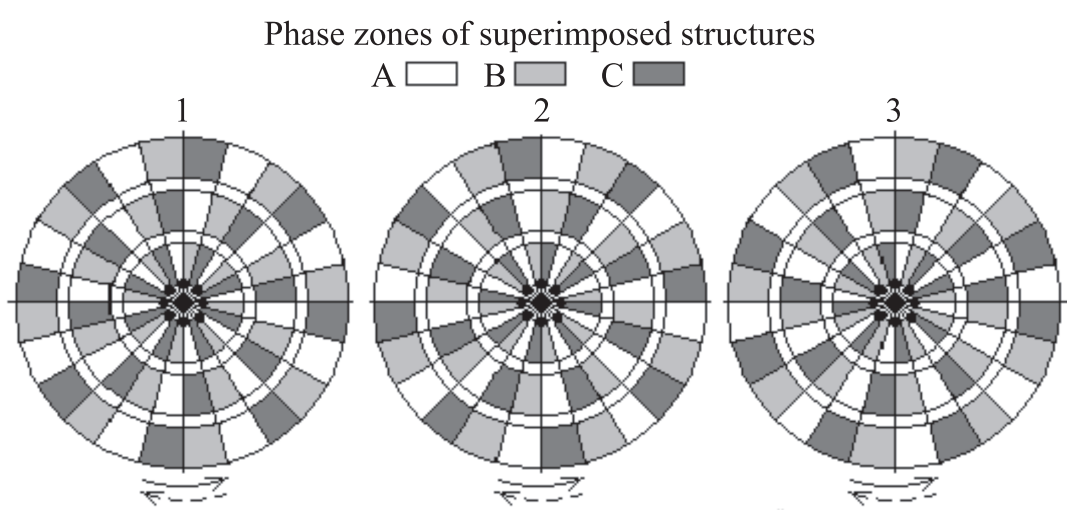

Tangential inversion of moving parts

Fig. 2. Axial-radial configuration $3(\mathrm{TF} 0.0 Y) \times(\mathrm{TF} 2.0 X)$.
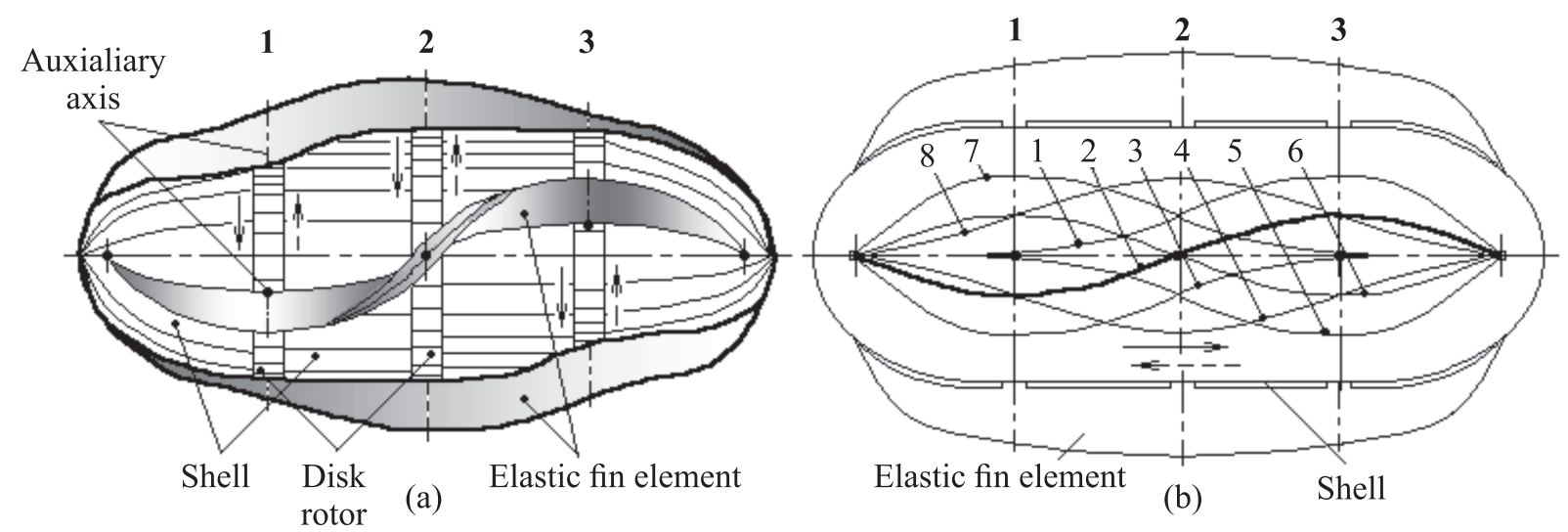

Fig. 3. Wave fin propulsion device: a) exterior view of the cylindrical shell; b) dynamics of fin elements.

reciprocating rotation. The wave inversion is achieved by inversion of the disk rotors on whose auxiliary axes the elastic fin elements are mounted.

The information and control system ensures the specified order of switching the current of structures 1, 2, 3 of the axial arrangement with simultaneous progressive switching of phases A, B, C of the radial arrangement. The synergetic effect leads to the synthesis of a traveling axial-radial magnetic field, which makes it possible to obtain the wave dynamics of the fin elements $[17,18]$.

An important goal of genetic engineering in electromechanics is to create generating types of parental chromosomes represented by an electromagnetic core of minimal mass, which are the basis for formation of a set of electromechanical structures ensuring the prescribed functions and efficiency of the electromechanical transducers (Fig. 4).

Let us consider the electromagnetic core of an inductor machine with two electric copper coils inside (Fig. 4a). One of the coils is an exciting coil with section $a \times b_{\mathrm{v}}$, and the other is an operating coil with section $a \times b_{\mathrm{p}}$.

To determine the conditions for obtaining the minimal mass of the electromechanical transducers of axial-radial configuration, it is necessary to solve three problems: determine the optimal copper/steel ratio; obtain the leading dimensions of the axial-radial configuration; establish the parameters of the electromechanical transducers of axial-radial configuration which give the minimal mass at the maximal capacity $[19,20]$. 


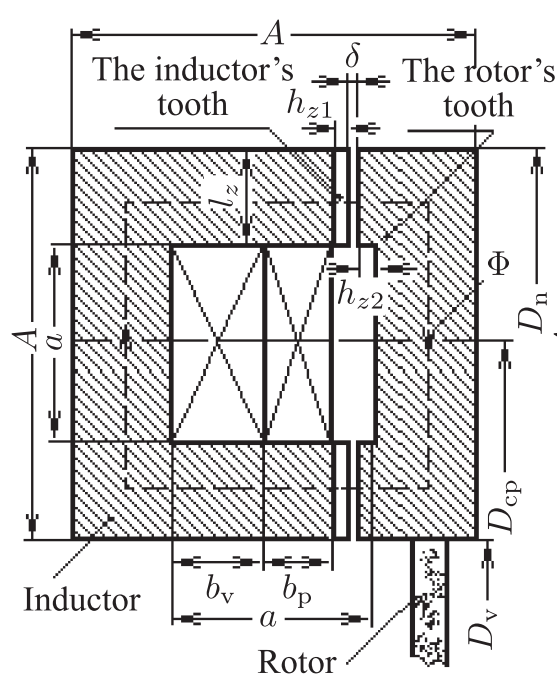

(a)

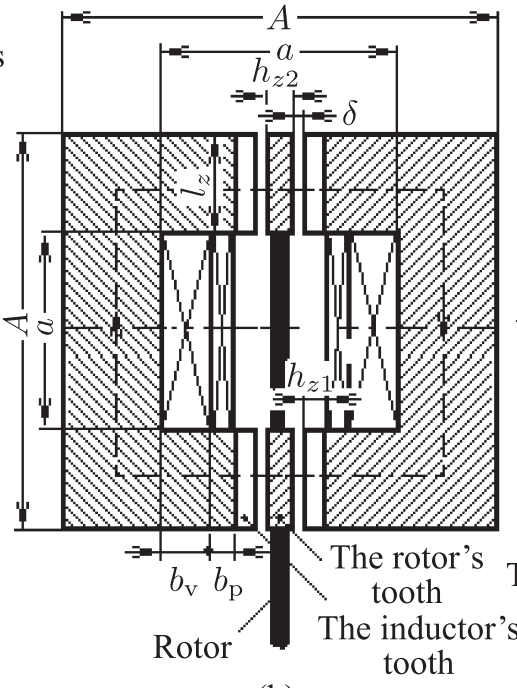

(b)

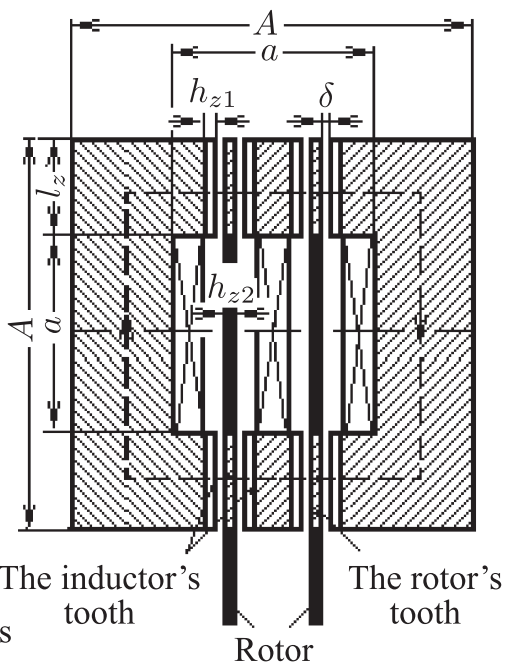

(c)

Fig. 4. Generating chromosomes represented by the section of the electromagnetic core: a) one inductor + one rotor; b) two inductors + one rotor; c) two inductor + two rotors.

\section{Optimal copper/steel ratios of the electromagnetic core}

The problem of optimization is to determine the ratios of the dimensions of the electromagnetic core at which the magnetic flux $F(a)$ attains the maximum [20, 21].

$$
\Phi(a)=\frac{\mu_{0} \mu j k_{\mathrm{z}} D_{\mathrm{cp}} a_{z}(A-a) a^{2}}{4\left(C_{2}+1\right)(A+a)}=C_{3} \frac{A-a}{A+a} a^{2},
$$

where $C_{2}=\Lambda_{c} / \Lambda_{\delta}$ is the ratio of the magnetic conductance of steel to that of the air gap; $C_{3}=\left(\pi D_{\mathrm{cp}} \mu_{0} \mu j k_{\mathrm{z}}\right) / 4\left(C_{2}+1\right)$ is a constant; $D_{\mathrm{cp}}$ is the average diameter of the magnetic system; $j$ is the current density in the coils; $k_{Z}$ is the coefficient of filling the window with the copper of the coils; $\alpha_{z}=b_{z} / t_{z}$ is the tooth overlap of the stator which is equal to the ratio of the width of the tooth $b_{z}$ to the slot pitch $t_{z}$.

Analysis of the function (1) for the maximum gives the equations

$$
\begin{gathered}
\frac{d \Phi}{d a}=C_{3} \frac{-2 a\left(a^{2}+A a-A^{2}\right)}{(A+a)^{2}}=0, \\
a^{2}+A a-A^{2}=0 .
\end{gathered}
$$

In the solution (3) the physical meaning has the positive root $a=-\frac{A}{2} \pm \frac{A}{2} \sqrt{5}$. This yields the conditions for the optimal copper/steel ratio

$$
a=0.61 A \quad \text { or } \quad A=1.61 a,
$$

which satisfies the radial height of the tooth of the inductor

$$
b_{z 1}=\frac{A-a}{2}=0.32 a
$$




\section{Determination of the leading dimensions of the electromechanical structures of axial-radial configuration}

The problem is solved by deriving the main design equation, taking into account the optimal copper/steel ratios (4)-(5) of the electromagnetic core [21, 22]. The section of the copper of the exciting coil is

$$
S_{\mathrm{v}}=\kappa_{\mathrm{pc}} \kappa_{\mathrm{zp}} k_{F} B_{\delta} \delta^{\prime} / \mu_{0} \kappa_{\mathrm{zm}} j,
$$

where $\kappa_{\mathrm{pc}}$ is the scattering coefficient of the magnetic flux; $\kappa_{\mathrm{zp}}$ is the safety factor for regulation of the generator's voltage; $k_{F}$ is the ratio of the magnetomotive force of the magnetic circuit to that of the gap; $B_{\delta}=B_{z 1} \kappa_{\mathrm{zc}} \alpha_{z}$ is the gap induction; $B_{z 1}$ is the admissible value of induction in the teeth of the inductor; $\kappa_{\mathrm{zc}}$ is the coefficient of filling with steel; $\delta^{\prime}=4(i-1) \delta$ is the calculated value of the air gap ( $i$ is the number of the axial layers or disks of the stator); $\mu_{0}=4 \pi \cdot 10^{-7} \mathrm{H} / \mathrm{m}$ is the magnetic constant; $\kappa_{\mathrm{zm}}$ is the coefficient of filling of the window with copper.

The internal diameter of the inductor is determined from the minimum permissible width of the tooth:

$$
D_{\mathrm{v}}=z b_{z} / \pi \alpha_{z}
$$

where $b_{z}=b_{z \min }=(3 \div 5) \cdot 10^{-3} \mathrm{~m}$ is the minimum permissible width of the tooth; $z=60 \mathrm{f} / \mathrm{n}$ is the number of pairs of the teeth of the inductor (rotor); $f$ is the current frequency, Hz; $n$ is the rotational velocity of the rotor, rev/min.

The section of the air gap for the optimal (5) dimension $l_{z}=0.32 a$ is

$$
S_{\delta}=\pi l_{z} D_{\mathrm{v}} \alpha_{z}=19.2 a b_{z} f / n .
$$

The electromotive force of the operating coils of the electromechanical structures of axialradial configuration is

$$
E=2.22 k_{\text {zp }} w f \Phi\left(k_{\lambda}-1\right) / k_{\lambda}
$$

where $k_{\lambda}=\lambda_{\max } / \lambda_{\min }$ is the modulation factor of the magnetic flux which is equal to the ratio of the maximal magnetic conductance $\lambda_{\max }$ to that of minimal $\lambda_{\min }$.

Under the assumption of the first harmonic of the magnetic flux, taking (9) into account, the air gap power can be written as

$$
P=E I_{\mathrm{n}}=2.22 k_{\mathrm{zp}} k_{\mathrm{zm}} j f S_{p} S_{\delta} B_{\delta}\left(\kappa_{\lambda}-1\right) / \kappa_{\lambda},
$$

where $I_{\mathrm{n}}=j S_{p}$ is the rated current of the operating coil.

The section of the copper of the operating coil is found from the expression (10):

$$
S_{p}=P \kappa_{\lambda} / 2.22\left(\kappa_{\lambda}-1\right) \kappa_{\mathrm{zp}} \kappa_{\mathrm{zm}} j f S_{\delta} B_{\delta},
$$

where $P=E I_{\mathrm{n}} \cos \varphi_{\mathrm{n}}$ is the design power; $\cos \varphi_{\mathrm{n}}$ is the rated power factor; $E=(1.15 \ldots 1.3) U_{\mathrm{n}}$ is the electromotive force for the generator and $E=(0.7 \ldots 0.85) U_{\mathrm{n}}$ is the electromotive force for the motor; $U_{\mathrm{n}}$ is the rated voltage.

Substituting (8) into (11), the section of the copper of the operating coil can be rewritten as

$$
S_{p}=\left[P \kappa_{\lambda} / 42.62\left(\kappa_{\lambda}-1\right) \kappa_{\mathrm{zp}} \kappa_{\mathrm{zm}} j a b_{z} B_{\delta}\right] \times\left(n / f^{2}\right) .
$$

The section of the window for the copper of the coils with the side $a$ is found as $a^{2}=S_{p}+S_{\mathrm{v}}$ or, taking (12) into account, as $a^{2}=c / a+S_{\mathrm{v}}$, where

$$
c=\left[P \kappa_{\lambda} / 42.62\left(\kappa_{\lambda}-1\right) \kappa_{\mathrm{zp}} \kappa_{\mathrm{zm}} j b_{z} B_{\delta}\right] \times\left(n / f^{2}\right)
$$

is the effective volume of the operating coil. 
This yields the main design equation, a cubic equation reduced to the incomplete form

$$
a^{3}-a S_{\mathrm{v}}-c=0 .
$$

In the case where $(c / 2)^{2}-\left(S_{\mathrm{v}} / 3\right)^{3} \geqslant 0$, one should use the Cardano solution, in which only the first real root has a physical meaning:

$$
a=\sqrt[3]{c / 2+\sqrt{(c / 2)^{2}-\left(S_{\mathrm{v}} / 3\right)^{3}}}+\sqrt[3]{c / 2-\sqrt{(c / 2)^{2}-\left(S_{\mathrm{v}} / 3\right)^{3}}} .
$$

Otherwise one has to resort to the trigonometric solution:

$$
a=2 \sqrt{\left(S_{\mathrm{v}} / 3\right) \cos (\alpha / 3)}
$$

where $\alpha=\arccos \left[c / 2 \sqrt{\left(S_{\mathrm{v}} / 3\right)^{3}}\right]$ is the constant defined by the parameters of the electric coils.

From (15)-(16) and (6), one determines the section of the operating coil

$$
S_{p}=a^{2}-S_{\mathrm{v}}
$$

and the dimensions of the other two sides of the exciting coil and the operating coil

$$
b_{\mathrm{v}}=S_{\mathrm{v}} / a ; \quad b_{p}=S_{p} / a .
$$

The leading dimensions (Fig. 4) are determined from the outer diameter

$$
D_{\mathrm{n}}=D_{\mathrm{v}}+A
$$

and the axial dimension

$$
l=(i-1)\left[2\left(h_{z 1}+\delta\right)+h_{z 2}\right]+i a+2 l_{z},
$$

where $i$ is the number of disks of the stator; $h_{z 1}$ and $h_{z 2}$ are the axial dimensions of the teeth of the inductor and the rotor, respectively.

Since the machine is designed so that the rated voltage, current, electric frequency and rotational velocity are taken to be given and the gap width, gap induction and current density can be chosen in accordance with design recommendations, the resulting relations (15)-(20) uniquely define the leading dimensions of the machine.

\section{Numerical simulation and minimization of the specific weight indices of axial-radial structures}

The total volume of electromechanical transducers is determined taking into account the outer diameter (19) and the axial dimension (20) of the machine:

$$
V=0.25 \pi D_{\mathrm{n}}^{2} l \text {. }
$$

The volume of the electromagnetic core is

$$
V_{\text {ea }}=V_{\mathrm{m}}+V_{\mathrm{ci}}+V_{\mathrm{cz}}+V_{\mathrm{cp}},
$$

where $V_{\mathrm{m}}=\pi D_{\mathrm{cp}} a^{2}$ is the coil copper volume; $V_{\mathrm{ci}}=N 3 z b_{\mathrm{i}} l_{z} a$ is the volume of the steel of the inductor; $V_{\mathrm{cz}}=N 2 z h_{z 1} b_{z 1} l_{z}$ is the volume of the steel of the inductor's teeth; $V_{\mathrm{cp}}=2 z h_{z 2} b_{z 2} l_{z}$ is the volume of the steel of the rotor's teeth; $D_{\mathrm{cp}}=0.5\left(D_{\mathrm{v}}+D_{\mathrm{n}}\right)$ is the average diameter of the inductor. 
The weight of the electromagnetic core is

$$
G_{\mathrm{a}}=V_{\mathrm{m}} g_{\mathrm{m}}+\left(V_{\mathrm{ci}}+V_{\mathrm{cz}}+V_{\mathrm{cp}}\right) g_{\mathrm{c}}
$$

where $g_{\mathrm{m}}$ and $g_{\mathrm{c}}$ are the specific weight indices of copper and steel, respectively.

The weight index of the electromagnetic core (23) per unit of the air gap power (10) is defined by the relation

$$
g_{\text {ea }}=G_{\mathrm{a}} / P .
$$

The numerical model of the electromechanical transducer of axial-radial configuration is based on the main design equation (14) and its solutions (15)-(16), which relate the design assignment to the geometry of the electromechanical transducer through optimal (4)-(5) copper/steel ratios of the electromagnetic core [21-23]. The calculation method involves step-bystep iteration computation. At the first stage, one calculates the electromagnetic cells, of which the elementary machine is composed (Fig. 5). This is done by calculating the section of the copper of the exciting coil (6), the operating coil (17) and the section of the window of the cell for copper and iron (4), (18). At the subsequent stages, the leading dimensions (19)-(20), volumes (21)-(22) and weight indices (23)-(24) are calculated.

At the beginning of calculation, the values of the airgap flux density and the current density of the coils, taken from the experience of designing inductor machines, are chosen as electromagnetic loads, and the design factors are introduced. Then the calculation is elaborated during the last iterations, in which the saturation of the magnetic circuit, the magnetic induction and the design factors obtained during the previous calculation operations are calculated. At the end of the calculation, a numerical solution of the field problem is obtained from the data on the geometry of the computational domain, and the electromagnetic calculation and the leading
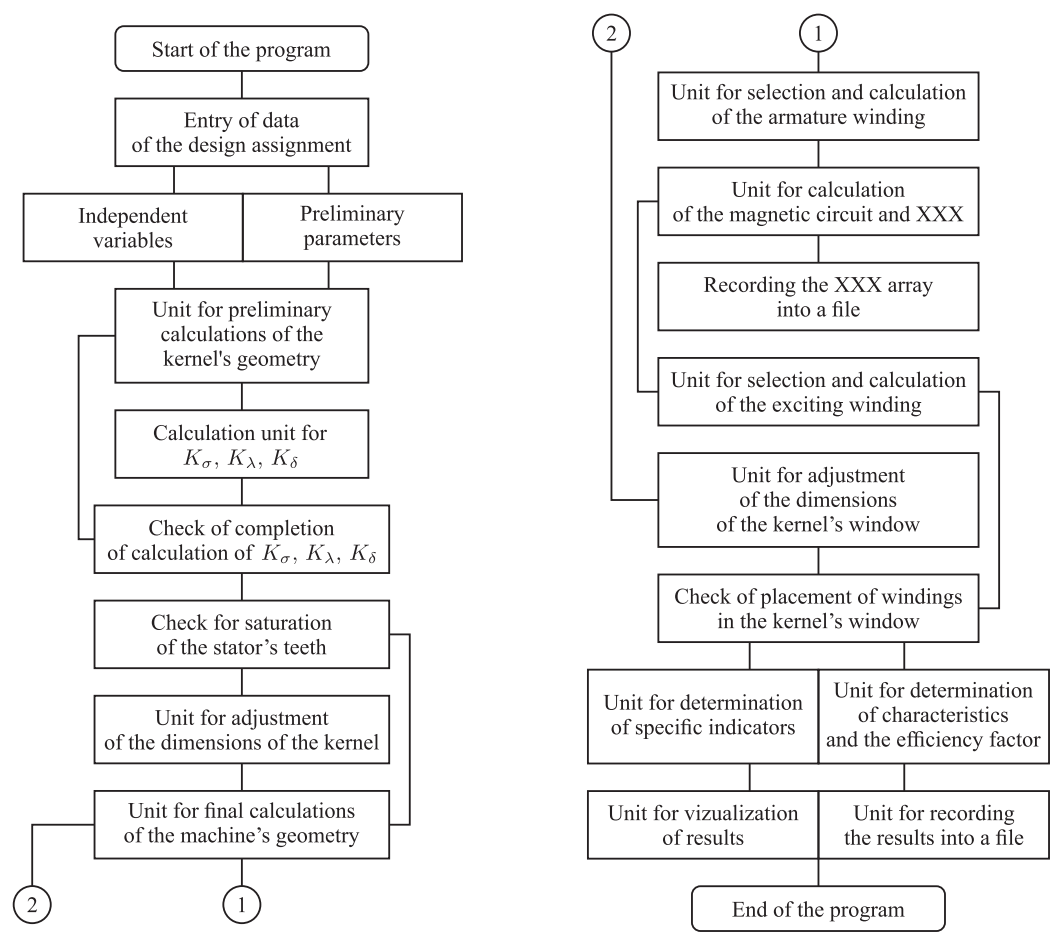

Fig. 5. The structure of the program for calculation of electromechanical transducers of axial-radial configuration. 
dimensions of the machine are elaborated. In the process of work, the location of the coils in the cell window area is checked, and, if necessary, its dimensions are adjusted. In the course of stepby-step iterations, the losses in copper and steel, the efficiency output and other characteristics of the machine are calculated by standard methods.

\section{Analysis of the results obtained and conclusions}

The genetic engineering techniques contribute to the development of various electromechanical structures which can be used in marine robotics. Electromechanical transducers of axial-radial configuration hold much promise as low-noise propulsion systems of marine robots. They make it possible to implement undulatory swimming modes used by hydrocoles.

Methods for developing new transducers of axial-radial configuration, which are based on the derivation of the main design equation, allow one to determine rational ratios of capacities, electric frequencies and rotational velocities to choose the leading dimensions of electromechanical transducers with minimal indices of specific weights. The design dependences of the overall dimension and the specific weight of the electromagnetic core of axial-radial configuration are a function of capacity, rotational velocity and electric frequency (Fig. 6).

For each capacity and frequency there exist minimal specific weights and the corresponding rational values of velocities. With increasing frequency and decreasing capacity the rational velocities move into the region of large values, and the range of rational velocities expands. To obtain the minimal materials consumption, it is necessary to select rational velocities corresponding to the minimal specific weights of the electromagnetic core and to choose its overall dimensions in the function of rational velocities (Fig. 7).

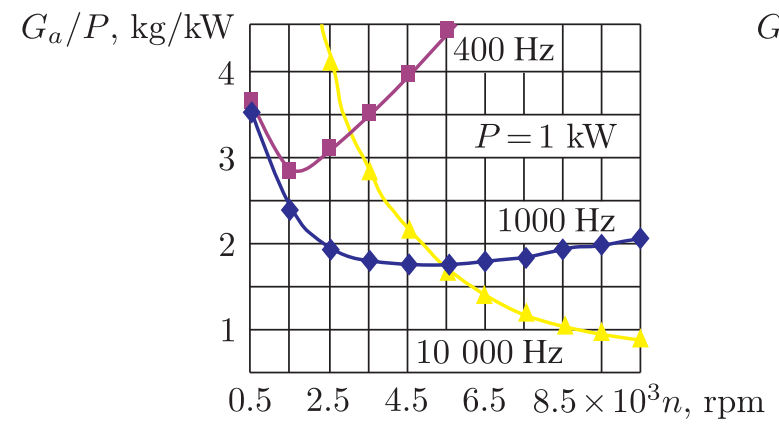

(a)

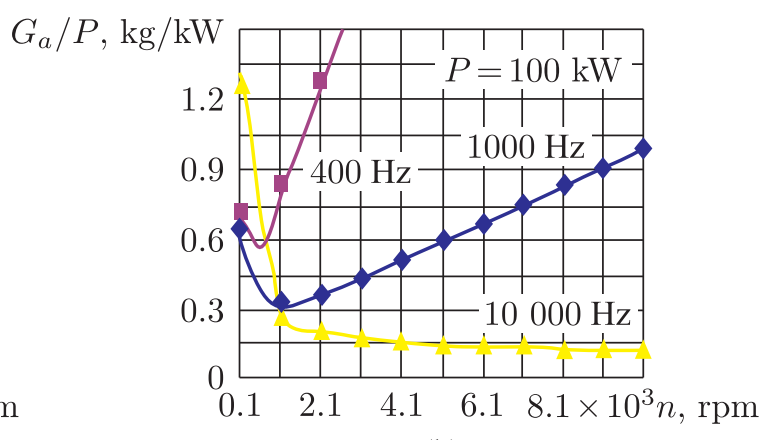

(b)

Fig. 6. Specific mass of the electromagnetic core: a) for $1 \mathrm{~kW}$; b) for $100 \mathrm{~kW}$.

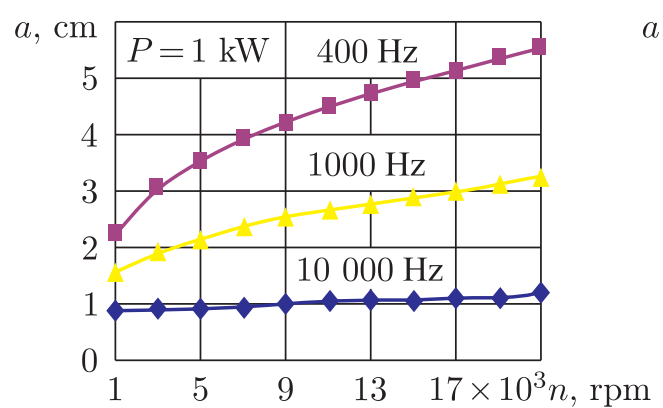

(a)

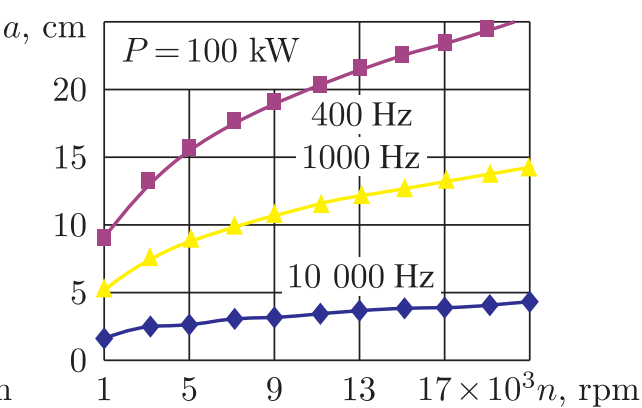

(b)

Fig. 7. Overall dimensions of the electromagnetic core: a) for $1 \mathrm{~kW}$; b) for $100 \mathrm{~kW}$. 
Compared to the conventional inductor machines with drum-type rotors, the weight of the active core of machines of axial-radial configuration can be reduced by a factor of 2.7 to 3.4 with a double decrease in volume. Electric machines of axial-radial configuration designed for frequencies of $50 \mathrm{~Hz}$ to $400 \mathrm{~Hz}$ are suitable for use in low-speed drives of wind and hydroelectric power plants and propulsion systems of underwater vehicles, and those designed for $1000 \mathrm{~Hz}$ to $10000 \mathrm{~Hz}$ can be used in high-speed drives of autonomous power plants of vehicles, aircraft, gyromotors, gas and steam-turbine plants.

\section{References}

[1] Antonelli, G., Underwater Robots, 3rd ed., Springer Tracts in Adv. Robot., vol.96, Springer, 2014,

[2] Slizheuski, B., Hydrobionics in Shipbuilding, Nikolaev: USMTU, 2002 (Russian).

[3] Lavars, N., Creepy Velox Robot Uses Undulating Fins to Skate over Solid Ice, https://newatlas.com/ice-skating-velox-robot/57587/ (2018).

[4] Barsukov, A.P., Components and Solutions for Creating Robots and Robotic Systems: No.2, Moscow: DMK-Press, 2005 (Russian).

[5] Bozhenov, Y. A. and Shamans, N. P., Hybrid Power Installation of Underwater Vehicles, Maritime Bull., 2012, no. 4(44), pp. 31-34.

[6] Franke, J., Kreitlein, S., Risch, F., and Guenther, S., Energy-Efficient Production Strategies and Technologies for Electric Drives, in IEEE Internat. Conf. on Industrial Technology (ICIT, Cape Town, 2013), pp. 1898-1903.

[7] Rulevskiy, V.M., Dementiev, Yu. N., and Bubnov, O.V., Power Supply Systems of RemoteControlled Underwater Vehicles, News of Tomsk Polytech. Univ., 2004, vol. 307, no. 5, pp. 120-123 (Russian).

[8] Shinkarenko, V.F., Principles of the Theory of Evolution of Electromechanical Systems, Kiev: Naukova Dumka, 2002 (Ukrainian).

[9] Shumilov, A.Yu., The Decade of the Monograph by V.F. Shinkarenko "Principles of the Theory of Evolution of Electromechanical Systems", Elektrotekh. i Elektromekh., 2012, no.3, pp.41-43 (Russian).

[10] Kuznietsov, Yu. and Shinkarenko, V., The Genetic Approach Is the Key to Innovative Synthesis of Complicated Technical Systems, J. Tech. Univ. Plovdiv, Bulgaria Fundament. Sci. Appl., 2011, vol. 16, no. 2, pp. 15-33.

[11] Gaidaicnko, J. and Shinkarenko, V., Principles of Structural Organisation and Genetic Creation Models of Hybrid Electromechanical Systems, in Proc. of the 11th Anniversary International Scientific Conference "Unitech'11" (Gabrovo, Bulgaria, Nov 2011): Vol. 1, pp. 79-84.

[12] Yakimovich, B. A., Korshunov, A. I., and Kuznetsov, A. P., Theoretical Bases of Structural and Technological Complexity of Products and Structures-Strategies of Industrial Systems of Mechanical Engineering, Izhevsk: UdGU, 2007 (Russian).

[13] Shaytor, N. M. and Ryaskov, Yu. I., Structural Synthesis of Magnetic Switching of Electromechanical Converters, in Proc. of the 12th Internat. Scientific Practical Conf. (2017), pp. 79-84.

[14] Shaytor, N. M., Afonin, A. A., and Ryaskov, Yu. I., Electromechanical System Axial-Radial Configurations, Ukrainian Patent 49630A, MKI 7 H02K19/18, H02K19/20, H02K19/10 (16.09.2002), no. 9, pp. 1-6.

[15] Shaytor, N. M., Gorpinchenko, A. V., and Rjaskoff, Y. I., Fin electropropulsion complex axial-radial configuration, Power Plants and Technologies, 2017, vol. 3, no. 4, pp. 82-88 (Russian).

[16] German-Galkin, S. G., Lebedev, V.V., and Bormotov, A.V., Modular Synchronous Inductor Machine in Electric Drive System, Mekhatronika, Avtomatizatsiya, Upravlenie, 2015, vol.16, no. 11, pp. 731-738 (Russian). 
[17] Afonin, A. A., The Problems of Converting the Configuration of the Electromechanical Energy Converters, Tekhn. Elektrodinam., 2001, no. 2, pp.49-53 (Russian).

[18] German-Galkin, S. G. and Bormotov, A. V., Analytical and Model Study of a Modular Electrical Machine in the Electrical Drive, Amer. J. Sci. Educ. Res., 2014, vol. 2, no. 1(4), pp. 614-625.

[19] Al'per, N. I. and Terzian, A. A., Inductor Generators, Moscow: Energia, 1970 (Russian).

[20] Shaytor, N. M., Features of Theory and Design Magnetoconductivity Electromechanical Transducers, Tekhn. Elektrodinam., 2002, no. 4, pp. 45-48 (Russian).

[21] Afonin, A. A., Shaytor, N. M., and Ryaskov, Yu. I., Calculation of Axial-Radial Inductor Generators Configuration, Tekhn. Elektrodinam., 2003, no.4, pp.41-45 (Russian).

[22] Shaytor, N. M., Problems and Prospects of Development of Electromechanical Transducers of New Technic Configurations, Tekhn. Elektrodinam., 2001, no. 1, pp. 55-59 (Russian).

[23] Ryaskov, Yu. I. and Shaytor, N. M., Induction Machines of Axial-Radial Configuration, Sevastopol: SevGU, 2018 (Russian). 